\title{
Infrared/terahertz spectra of the photogalvanic effect in (Bi,Sb)Te based three-dimensional topological insulators
}

\author{
H. Plank, ${ }^{1}$ J. Pernul, ${ }^{1}$ S. Gebert, ${ }^{1}$ S. N. Danilov,${ }^{1}$ J. König-Otto, ${ }^{2}$ S. Winnerl, ${ }^{2}$ M. Lanius, ${ }^{3}$ J. Kampmeier, ${ }^{3}$ G. Mussler, ${ }^{3}$ \\ I. Aguilera, ${ }^{4}$ D. Grützmacher, ${ }^{3}$ and S. D. Ganichev ${ }^{1, *}$ \\ ${ }^{1}$ Terahertz Center, University of Regensburg, 93051 Regensburg, Germany \\ ${ }^{2}$ Helmholtz Zentrum Dresden, 01328 Rossendorf, Germany \\ ${ }^{3}$ Peter Grünberg Institute (PGI-9) \& Jülich Aachen Research Alliance (JARA-FIT), 52425 Jülich, Germany \\ ${ }^{4}$ Peter Grünberg Institute (PGI-1) \& Institute for Advanced Simulation (IAS-1), Forschungszentrum Jülich and JARA, 52425 Jülich, Germany
}

(Received 30 November 2017; published 16 February 2018)

\begin{abstract}
We report on the systematic study of infrared/terahertz spectra of photocurrents in (Bi,Sb)Te based threedimensional topological insulators. We demonstrate that in a wide range of frequencies, ranging from fractions up to tens of terahertz, the photocurrent is caused by the linear photogalvanic effect (LPGE) excited in the surface states. The photocurrent spectra reveal that at low frequencies the LPGE emerges due to free carrier Drude-like absorption. The spectra allow us to determine the room temperature carrier mobilities in the surface states despite the presence of thermally activated residual impurities in the material bulk. In a number of samples we observed an enhancement of the linear photogalvanic effect at frequencies between 30 and $60 \mathrm{THz}$, which is attributed to the excitation of electrons from helical surface to bulk conduction band states. Under this condition and applying oblique incidence we also observed the circular photogalvanic effect driven by the radiation helicity.
\end{abstract}

DOI: 10.1103/PhysRevMaterials.2.024202

\section{INTRODUCTION}

Three-dimensional topological insulators (TIs) caught attention soon after their prediction, for reviews see Refs. [1-3]. The band structure at the surface is the reason for their unique features: alike to graphene [4], the surface states of topological insulators are characterized by a linear energy dispersion, which is described by the zero mass Dirac equation. The single Dirac cone in TIs, however, leads to a spin-momentum locking and, with that, to new physics. Renowned techniques such as angle resolved photoemission spectroscopy (ARPES) $[1-3,5]$ or magnetotransport measurements, for review see Ref. [6], are applied to access and characterize surface carriers in TIs. New opportunities to study Dirac fermions are offered by nonlinear high frequency transport phenomena [7] which scale with the second or third power of radiation electric field. A plethora of such effects has been theoretically discussed and observed in TIs systems including circular and linear photogalvanic effects in three-dimensional (3D) TIs [8-19], edge photogalvanics in two-dimensional (2D) TIs [20-23], quantum interference controlled photocurrents [24,25], ultrafast photocurrents in TI states [26-30], transient photocurrents in the topological surface state measured by ARPES and its modifications [31-34], the inverse spin-galvanic effect [35], and harmonic generation [36-38], for review see Ref. [39]. The advantage is that some of them can be used to excite solely the surface states even in TI materials with a high carrier density in the bulk and even at room temperature.

\footnotetext{
*Corresponding author: sergey.ganichev@physik.uni-regensburg. de
}

In this paper we present a systematic study of the photogalvanic effect in a wide frequency range extending over two orders of magnitude from $f \approx 0.6$ to $60 \mathrm{THz}$. The experiments were carried out on various $(\mathrm{Bi}, \mathrm{Sb}) \mathrm{Te}$ based $3 \mathrm{D}$ TIs at room temperature. The samples, besides their composition, discriminate due to their Fermi level position or bulk carrier concentration. For low frequency radiation and normal incidence the photocurrent is caused by the linear photogalvanic effect (LPGE). The spectra measured reveal that they follow the Drude high-frequency conductivity varying with the radiation frequency as $1 /\left[1+(2 \pi f \tau)^{2}\right]$, where $f$ is the radiation frequency and $\tau$ is the scattering time of surface states carriers. These results are analyzed applying the microscopic theory developed in Refs. [11,17] and provide an access to the room temperature scattering times and mobilities of the surface states. In some samples we observed a resonancelike increase of the LPGE at high frequencies in the range from 30 to $60 \mathrm{THz}$. The enhancement of the LPGE is attributed to the photoionization of Dirac fermions in the surface states to the conduction/valence band. We discuss the microscopic model of this phenomenon and show that the photocurrent is formed by a shift contribution or an asymmetric relaxation of the photoexcited electrons/holes. Furthermore, in this frequency range and applying oblique incidence, apart from the LPGE, we also observed a circular photogalvanic effect driven by the radiation helicity.

\section{SAMPLES AND TECHNIQUE}

For this study, we used molecular beam epitaxy (MBE) grown $\left(\mathrm{Bi}_{1-x} \mathrm{Sb}_{x}\right)_{2} \mathrm{Te}_{3}$ based 3D TIs including a pure $\mathrm{Bi}_{2} \mathrm{Te}_{3}$ sample $[40,41], \mathrm{Bi}_{2} \mathrm{Te}_{3} / \mathrm{Sb}_{2} \mathrm{Te}_{3}$ heterostructures with different 
TABLE I. Samples compositions, Fermi energies $E_{\mathrm{F}}$ and Fermi velocities $v_{\mathrm{F}}$, together with scattering times $\tau$ obtained from the frequency dependencies of the linear photogalvanic effect. The scattering times are extracted with a tolerance value of $10 \%$. The corresponding values of the carrier mobilities $\mu$ are calculated from the scattering times and the Fermi energies $E_{\mathrm{F}}$. Note that the latter values are measured by in situ ARPES and, in particular for $\left(\mathrm{Bi}_{0.06} \mathrm{Sb}_{0.94}\right)_{2} \mathrm{Te}_{3}$ with $E_{\mathrm{F}}$ close to zero, may yield overestimated values of $\mu$, see Ref. [67].

\begin{tabular}{|c|c|c|c|c|c|c|}
\hline \multirow[b]{2}{*}{ Sample } & \multirow[b]{2}{*}{$\mathrm{Bi}_{2} \mathrm{Te}_{3}$} & \multicolumn{3}{|c|}{$\mathrm{Bi}_{2} \mathrm{Te}_{3} / \mathrm{Sb}_{2} \mathrm{Te}_{3}$} & \multicolumn{2}{|c|}{$\left(\mathrm{Bi}_{1-x} \mathrm{Sb}_{x}\right)_{2} \mathrm{Te}_{3}$} \\
\hline & & $d_{\mathrm{ST}}=7.5 \mathrm{~nm}$ & $d_{\mathrm{ST}}=15 \mathrm{~nm}$ & $d_{\mathrm{ST}}=25 \mathrm{~nm}$ & $x=0.43$ & $x=0.94$ \\
\hline$E_{\mathrm{F}}(\mathrm{meV})$ & 500 & 140 & 30 & -35 & 500 & 7 \\
\hline$v_{\mathrm{F}}\left(10^{5} \mathrm{~m} / \mathrm{s}\right)$ & 4.3 & 5.2 & 2.2 & 2.5 & 5.1 & 3.8 \\
\hline$\tau(\mathrm{ps})$ & $>0.25$ & 0.06 & 0.06 & 0.08 & $>0.25$ & 0.04 \\
\hline$\mu\left(\mathrm{cm}^{2} / \mathrm{Vs}\right)$ & $>940$ & 1230 & 1030 & 1420 & $>1330$ & 8210 [67] \\
\hline
\end{tabular}

thicknesses of the $\mathrm{Sb}_{2} \mathrm{Te}_{3}$ layer $[42,43]$, and $\left(\mathrm{Bi}_{1-x} \mathrm{Sb}_{x}\right)_{2} \mathrm{Te}_{3}$ ternary systems [44,45]. The samples, grown on a $(111)$ oriented silicon substrate, were characterized by in situ ARPES measurements, from which the energy dispersion and the Fermi level position were obtained. All samples compositions, Fermi velocities, and energies with respect to the Dirac point are summarized in Table I. The latter varies from -35 up to $500 \mathrm{meV}$. In the ternary systems ARPES measurements showed that in the ternary alloy with an Antimony concentration $x=0.94$ the Fermi energy $E_{\mathrm{F}}$ lies close to the Dirac point. Note that a reduced bulk carrier concentration is found at $x=0.43$, see Refs. $[44,45]$. In the heterostructures, where $\mathrm{Sb}_{2} \mathrm{Te}_{3}$ layers are grown on a $10 \mathrm{~nm} \mathrm{Bi}_{2} \mathrm{Te}_{3}$ layer, the Fermi level is tunable by varying the $p$-type $\mathrm{Sb}_{2} \mathrm{Te}_{3}$ thickness $d_{\mathrm{ST}}$, see Refs. [42,43]. $\mathrm{X}$-ray diffraction (XRD) measurements were performed to confirm the alignment of the substrate with the thin TI layer and to determine the in-plane crystallographic axes. With knowledge of the latter, the samples were cut along directions of high symmetry into $7 \times 4 \mathrm{~mm}^{2}$ pieces. The samples were electrically contacted in the middle of the edges parallel to the $x$ and $y$ directions, see insets in Figs. 1(a) and 1(b).

To cover a wide range of frequencies numerous sources of continuous wave (cw) and pulsed infrared/terahertz laser radiation were applied including optically pumped molecular

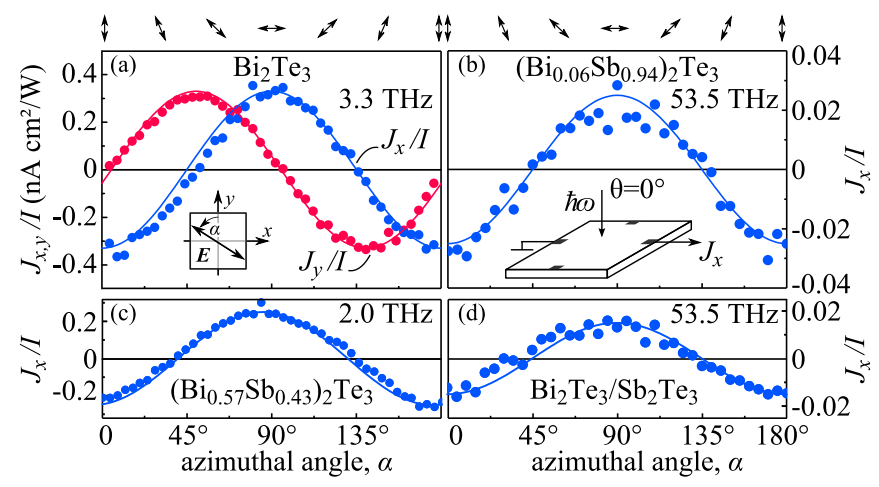

FIG. 1. Photocurrent $J_{x, y}$ measured along $x$ and $y$ directions and normalized on the radiation intensity $I$ in (a) $\mathrm{Bi}_{2} \mathrm{Te}_{3}$, (b) $\left(\mathrm{Bi}_{0.06} \mathrm{Sb}_{0.94}\right)_{2} \mathrm{Te}_{3}$, (c) $\left(\mathrm{Bi}_{0.57} \mathrm{Sb}_{0.43}\right)_{2} \mathrm{Te}_{3}$, and (d) $\mathrm{Bi}_{2} \mathrm{Te}_{3} / \mathrm{Sb}_{2} \mathrm{Te}_{3}$ heterostructure with $\mathrm{Sb}_{2} \mathrm{Te}_{3}$ thickness of $15 \mathrm{~nm}$. Solid lines show fit after Eq. (1), see also Eq. (4) and discussion. Note that the polarization independent offset $D\left(D^{\prime}\right)$, being much smaller than the amplitude $A(f)$, is subtracted in these plots. The insets in panels (a) and (b) define the angle $\alpha$ and show the experimental setup. Arrows on top illustrate the polarization plane orientation for several angles $\alpha$. terahertz lasers $[46,47]$, free electron lasers (FELBE) at the Helmholtz-Zentrum Dresden-Rossendorf $[48,49]$, a quantum cascade laser (QCL) [50,51] as well as $Q$-switched and transversely excited atmospheric pressure (TEA) $\mathrm{CO}_{2}$ lasers [52-54]. The lasers operated at single frequencies in the range from $f \approx 0.6$ to $60 \mathrm{THz}$ (corresponding photon energies range from $\hbar \omega=2.5$ to $250 \mathrm{meV}$, where $\omega=2 \pi f$ is the angular frequency). For the low frequency range from 0.6 to $3 \mathrm{THz}$ a line-tunable pulsed molecular laser was used with $\mathrm{CH}_{3} \mathrm{~F}$, $\mathrm{D}_{2} \mathrm{O}$, and $\mathrm{NH}_{3}$ as active media $[55,56]$. The laser generated single pulses with a duration of about $100 \mathrm{~ns}$ with a repetition rate of $1 \mathrm{~Hz}$. The radiation intensity on the sample surface was about $10 \mathrm{~kW} / \mathrm{cm}^{2}$. Furthermore, low frequency measurements were performed in the range from $f=1.8$ to $10 \mathrm{THz}$ with the tunable free electron laser FELBE 1 (U-27) operating in the quasi $c w$ regime. The FELBE provided picosecond micropulses with repetition rates in the $\mathrm{MHz}$ range and an average power of tens of $\mathrm{mW}$.

Radiation with frequencies of about $30 \mathrm{THz}$ was obtained by pulsed line-tunable $Q$-switched and TEA $\mathrm{CO}_{2}$ lasers. The Q-switched laser provided pulse durations of hundreds of nanoseconds with a peak power of about $1 \mathrm{~kW}$ and a repetition rate of about $120 \mathrm{~Hz}$ [54]. The operation mode of the TEA $\mathrm{CO}_{2}$ lasers $[47,52]$ was similar to the one of the molecular terahertz lasers. Further lines in this range and at higher frequencies up to $60 \mathrm{THz}$ were obtained applying the free electron laser FELBE 2 (U-100), operating in the same regime as FELBE 1 described above. Radiation with $f=58 \mathrm{THz}$ was additionally provided by a $c w$ quantum cascade laser with a power of about $10 \mathrm{~mW}$.

The peak power of the radiation was monitored, depending on the system, with mercury cadmium telluride (MCT) [57] and photon-drag [58] detectors, as well as with pyroelectric power meters. The beam positions and profiles were checked with pyroelectric cameras $[59,60]$ or thermal sensitive paper. The radiation was focused onto spot sizes of about 1 to $4 \mathrm{~mm}$ diameter, depending on the radiation frequency. Experimental geometry included normal as well as oblique incidence. In experiments at normal incidence, front and back illumination was used with corresponding angles of incidence $\theta=0$ and $180^{\circ}$, see inset in Fig. 1(b). The back illumination was used to ensure that the signal is caused by the linear photogalvanic effect, being the focus of this work, and to ensure that there is only a negligible contribution of the photon drag effect [17], which, if present, can affect the frequency dependence of the photocurrent. In the measurements applying oblique incident radiation, aimed at the search for the circular photogalvanic 
effect [61] in 3D TI at terahertz frequencies [10], the angle of incidence $\theta$ was varied between $-40^{\circ}$ and $40^{\circ}$ with the $(y z)$ plane of incidence, see inset in Fig. 4(b).

In the majority of the experiments, linearly polarized radiation with an azimuthal angle $\alpha$ defining the orientation of the radiation electric field vector in the sample's plane and the $y$ axis, see inset in Fig. 1(a), was applied. The angle $\alpha$ was varied either by rotation of half-wave plates or a grid wire placed behind a quarter-wave Fresnel rhomb, which was set to provide circularly polarized radiation. To study the helicity dependence of the signal quarter-wave Fresnel rhombs or plates were used. In this geometry, the radiation helicity was varied as $P_{\text {circ }} \propto \sin 2 \varphi$, where the rotation angle $\varphi$ was defined as an angle between the laser polarization plane and the optical axis of the polarizers [62,63]. Note that for $\alpha=0$ as well as for $\varphi=0$ the incident radiation was linearly polarized with electric field vector parallel to the $y$ axis. The induced photocurrents were detected as a voltage drop across load resistors at room temperature. The signals were recorded either with $\mathrm{GHz}$ oscilloscopes, in the case of pulsed gas laser systems, or with lock-in technique, in the case of modulated quasi $c w$ radiation of FELBE and $c w$ radiation of the QCL. The photocurrents were measured in two directions, $x$ and $y$, perpendicular to each other and parallel to the sample edges, see insets in Fig. 1.

\section{EXPERIMENTAL RESULTS}

A photocurrent excited by normal incident linearly polarized radiation was detected for all used frequencies and samples. It is characterized by the same overall behavior: It scaled quadratically with the radiation electric field, had a response time of picoseconds or less, and exhibited a characteristic polarization dependence. Figure 1(a) presents an example of the photocurrent variation upon rotation of the radiation polarization plane obtained in $\mathrm{Bi}_{2} \mathrm{Te}_{3}$ excited with radiation frequency $f=3.3 \mathrm{THz}$. The figure shows that the photocurrent scales after

$$
\begin{aligned}
& J_{x}(\alpha) / I=A(f) s_{1}+D(f), \\
& J_{y}(\alpha) / I=-A(f) s_{2}+D^{\prime}(f),
\end{aligned}
$$

where $s_{1}=-\cos 2 \alpha$ and $s_{2}=-\sin 2 \alpha$ are the Stokes parameters of light defining the electric field orientation in the $x, y$ coordinate system and in a $45^{\circ}$ rotated one, respectively $[64,65]$. Note that in all experiments the polarization independent offset $D(f)$ and $D^{\prime}(f)$ was much smaller than $A(f)$, and therefore, is out of the scope of this paper. Figures 1(b)-1(d) show exemplary $J_{x}(\alpha)$ measured for further three samples including $\left(\mathrm{Bi}_{1-x} \mathrm{Sb}_{x}\right)_{2} \mathrm{Te}_{3}$ ternaries with two different $\mathrm{Sb}$ concentrations $x$ and one of the $\mathrm{Bi}_{2} \mathrm{Te}_{3} / \mathrm{Sb}_{2} \mathrm{Te}_{3}$ heterostructures. Experiments with front and back illumination demonstrated that the polarization dependence itself, and sign and value of the coefficients $A(f)$ do not change. This result was the same in all samples and for all frequencies used in this work (data not shown). The dependence of the coefficient $A(f)$ on the frequency is shown in Fig. 2. The data reveal that in a wide range of frequencies the photocurrent decreases with the frequency increase and can be well fitted by

$$
A(f) \propto 1 /\left[1+(2 \pi f \tau)^{2}\right] .
$$

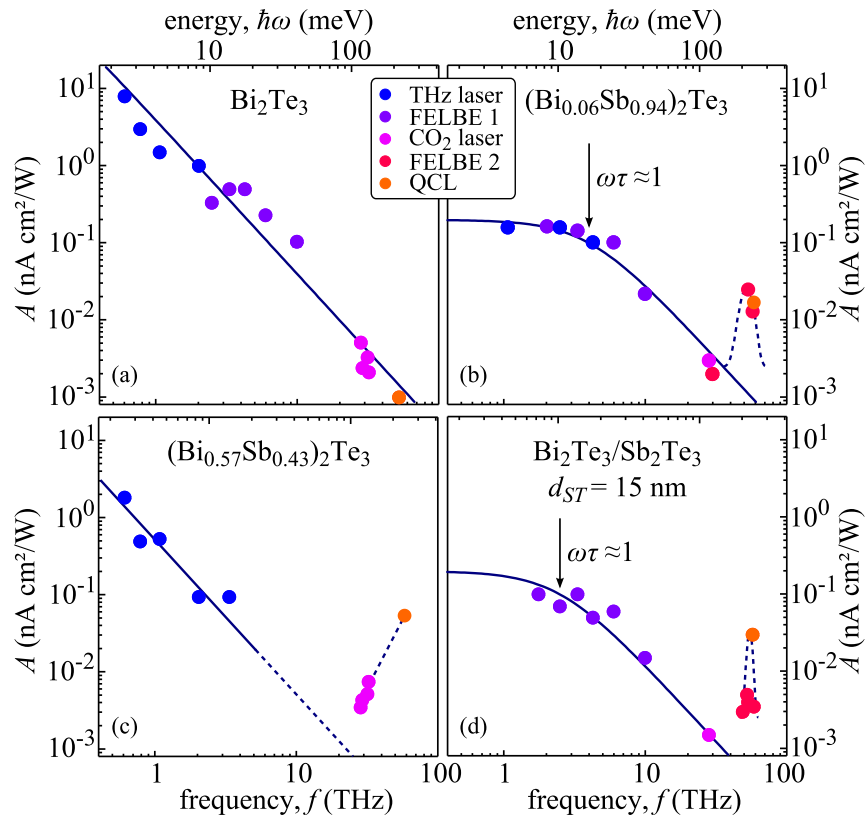

FIG. 2. Frequency dependence of the coefficient $A$ for (a) $\mathrm{Bi}_{2} \mathrm{Te}_{3}$, (b) $\left(\mathrm{Bi}_{0.06} \mathrm{Sb}_{0.94}\right)_{2} \mathrm{Te}_{3}$, (c) $\left(\mathrm{Bi}_{0.57} \mathrm{Sb}_{0.43}\right)_{2} \mathrm{Te}_{3}$, and (d) $\mathrm{Bi}_{2} \mathrm{Te}_{3} / \mathrm{Sb}_{2} \mathrm{Te}_{3}$ heterostructure with $\mathrm{Sb}_{2} \mathrm{Te}_{3}$ thickness of $15 \mathrm{~nm}$. Solid line shows fit after Eq. (2), see also Eq. (6) and discussion. Dashed lines are guide for eye, demonstrating deviation of photocurrent amplitude from the Drude-like behavior.

At high frequencies, however, a substantial deviation from this behavior has been detected for several samples: The photocurrent drastically increases as compared to the values expected from Eq. (2), reaches a maximum, and then decreases, see Figs. 2(b), 2(d) and 3(a). Note that, while the used discrete frequencies clearly indicate the photocurrent enhancement, they do not allow a characterization of the peak with a satisfactory resolution. As addressed above, the overall behavior at these frequencies including its polarization and angle of

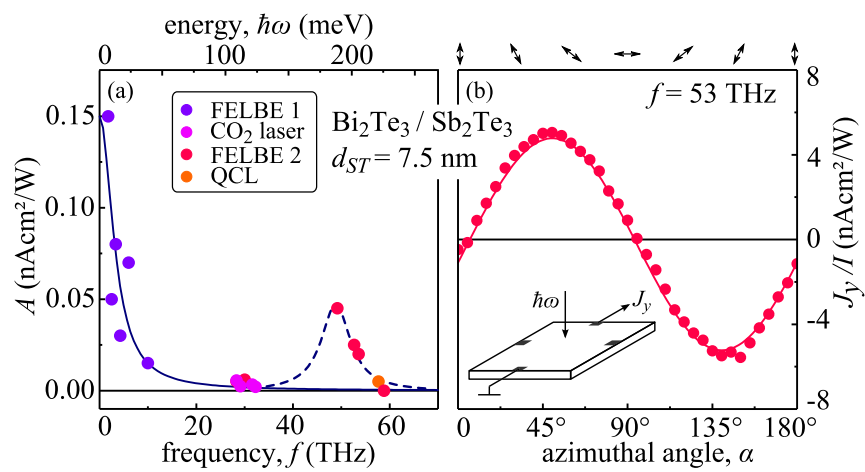

FIG. 3. (a) Frequency dependence of coefficient $A$ of a $\mathrm{Sb}_{2} \mathrm{Te}_{3} / \mathrm{Bi}_{2} \mathrm{Te}_{3}$ heterostructure with $d_{\mathrm{ST}}=7.5 \mathrm{~nm}$. Solid line shows fit after Eq. (2), see also Eq. (6) and discussion. Dashed line is guide for eye, demonstrating deviation of photocurrent amplitude from the Drude-like behavior. (b) Azimuthal angle dependence of the photocurrent $J_{y} / I$ measured at frequency $f=53 \mathrm{THz}$. Solid lines show fit after Eq. (1), see also Eq. (4) and discussion. Inset shows experimental setup. 


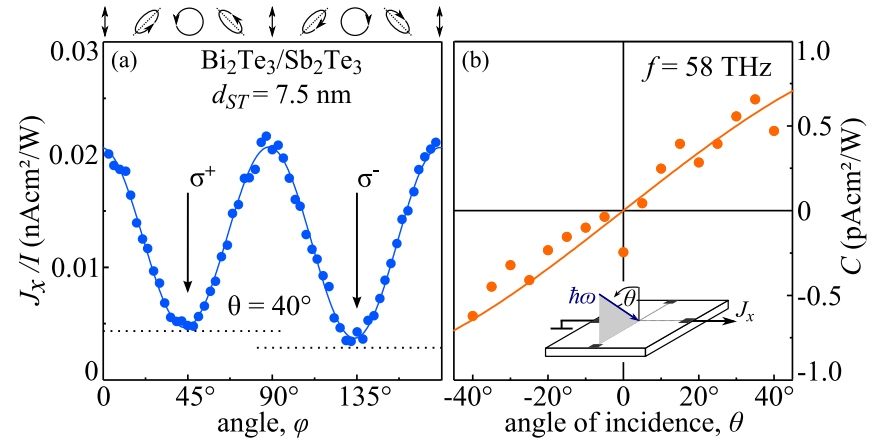

FIG. 4. (a) Dependence of the normalized photocurrent $J_{x} / I$ on the angle $\varphi$ measured in a $\mathrm{Sb}_{2} \mathrm{Te}_{3} / \mathrm{Bi}_{2} \mathrm{Te}_{3}$ heterostructure with $d_{\mathrm{ST}}=7.5 \mathrm{~nm}$. Data are shown for the $(y z)$ plane of incidence and angle of incidence $\theta=40^{\circ}$. Solid line shows fit after Eq. (3), see also Eq. (7) and discussion. Horizontal lines and downwards pointing arrows indicate photocurrent for circularly polarized radiation. (b) Dependence of the circular coefficient $C$ on the angle of incidence. Solid line shows fit after Eq. (3), see also Eq. (7) and discussion. Inset shows experimental setup.

incidence dependencies remained unchanged, as shown in Figs. 1(b), 1(d) and 3(b).

For oblique incidence and linearly polarized radiation we observed the same dependence $J(\alpha)$, however, the amplitude $A(f)$ depending on sample and radiation frequency decreased or increased (data not shown, for the origin see Ref. [17]). Using elliptically polarized radiation, however, we observed that in the direction normal to the plane of incidence the polarization dependence was modified. Besides the LPGE, varying after $J_{x}(\varphi)=-A(f, \theta)(\cos (4 \varphi)+1) / 2=$ $A(f, \theta) s_{1}$, we observed a small but clearly pronounced additional photocurrent contribution, which has opposite signs for right- and left-handed circularly polarized radiation. The overall polarization dependence in this geometry is well described by

$$
J_{x}(\varphi) / I=A(f, \theta) s_{1}+C(f, \theta) s_{3}+D(f, \theta),
$$

see Fig. 4(a). The observed circular photocurrent is proportional to the coefficient $C(f, \theta)$ and the Stokes parameter $s_{3}=\sin (2 \varphi)$ defining the radiation helicity $[64,65]$. Figure 4(b) shows $C(f, \theta)$ as a function of the angle of incidence $\theta$, revealing that it is odd in $\theta$.

\section{DISCUSSION}

First, we discuss the data obtained at normal incidence. According to the symmetry analysis, spatially homogeneous normal incident radiation can result in the photogalvanic effect in the surface states as well as the photon drag effect $[11,17]$. The corresponding current density $\boldsymbol{j}$ is given by

$$
\begin{aligned}
& j_{x}=\left(\chi+\mathcal{T} q_{z}\right)\left[\left|E_{x}\right|^{2}-\left|E_{y}\right|^{2}\right], \\
& j_{y}=-\left(\chi+\mathcal{T} q_{z}\right)\left[E_{x} E_{y}^{*}+E_{y} E_{x}^{*}\right] .
\end{aligned}
$$

Here $E_{x, y}$ are in-plane projections of the radiation electric field $\boldsymbol{E}$, the factor $\chi$ is the single linearly independent photogalvanic constant, $\mathcal{T}$ is the photon drag constant, $\boldsymbol{q}$ is the photon wave vector, and $z$ is normal to the epilayer. Note that the squared brackets in Eq. (4) divided by the electric field amplitude $\left|E_{0}\right|^{2}$ represent the Stokes parameters $s_{1}$ and $s_{2}$, see Refs. $[64,65]$. These characteristic polarization dependencies have been observed for all samples and frequencies used, see Figs. 1 and 3(b).

The fact that in the experiments the photocurrent amplitude $A(f)$ remains unchanged for front and back illumination excludes the sizable contribution of the photon drag effect and provides a clear evidence for the photogalvanic effect in the surface states as a cause of the photocurrent $[11,17]$. Indeed, the photogalvanic effect is determined only by the in-plane electric field orientation, see Eq. (4), and is insensitive to the radiation propagation direction. The photon drag current on the other hand, being proportional to the photon momentum, reverses its sign at inversion of the photon wave vector $q_{z}$, see also Eq. (4). Therefore, a substantial contribution of the photon drag effect should result either in different magnitudes $A(f)$ for front and back excitation or, if dominating, even in a change of the photocurrent direction.

For small photon energies, at which Drude absorption dominates [66] and direct optical transitions are not possible, the linear photogalvanic effect is shown to be caused by the asymmetric scattering of Dirac fermions driven back and forth by the terahertz electric field $[11,17]$. For elastic scattering by Coulomb impurities the photogalvanic coefficient $\chi$ in Eq. (4) is given by [11]

$$
\chi=e v_{\mathrm{F}} \frac{2 \tau}{E_{\mathrm{F}}} \Xi \sigma(f)
$$

in which $e$ is the electric charge, $\Xi$ the asymmetric scattering probability, and $\sigma(f)$ is the high frequency (Drude) conductivity given by

$$
\sigma(f)=\frac{e^{2} E_{\mathrm{F}} \tau}{4 \pi \hbar^{2}\left[1+(2 \pi f \tau)^{2}\right]} .
$$

Equations (5) and (6) reveal that the amplitude of the photocurrent should scale after Eq. (2). Our measurements performed in a wide frequency range, apart from the resonantlike increase at high frequencies observed in some samples, confirm this frequency dependence, see Figs. 2 and 3. Consequently, the investigation of the photogalvanic effect allows one to analyze the Drude conductivity of the surface states, which provides access to the scattering times. In our data the latter one can be extracted for $\left(\mathrm{Bi}_{0.06} \mathrm{Sb}_{0.94}\right)_{2} \mathrm{Te}_{3}$ and $\mathrm{Bi}_{2} \mathrm{Te}_{3} / \mathrm{Sb}_{2} \mathrm{Te}_{3}$ heterostructures with different thicknesses of $\mathrm{Sb}_{2} \mathrm{Te}_{3}$. For these samples the condition $\omega \tau \approx 1$ is fulfilled, see Figs. 1(b) and 1(d), in the studied frequency range. The values of $\tau$ can be estimated from the fit functions and are summarized in Table I. Taking into account the Fermi level position, known from in situ ARPES (see Table I), we obtained room temperature mobilities of the Dirac states in these samples ranging from $1000 \mathrm{~cm}^{2} /$ Vs up to several thousands [67]. These are of the same order of magnitude as the ones measured in transport experiments for low temperature from 2 up to $77 \mathrm{~K}$, see, e.g., Refs. [45,68].

The frequency dependencies of the photocurrent obtained for $\mathrm{Bi}_{2} \mathrm{Te}_{3}$ and $\left(\mathrm{Bi}_{0.57} \mathrm{Sb}_{0.43}\right)_{2} \mathrm{Te}_{3}$ show that the photocurrent scales as $1 / f^{2}$ down to the lowest frequencies used here, see Figs. 2(a) and 2(c). This indicates that the value $\omega \tau$ remains 
substantially larger than unity. Consequently, the scattering times in these samples are at least by one order of magnitude larger than that for previously discussed samples. To determine $\tau$ in these samples further measurements with substantially lower frequencies at which $\omega \tau$ becomes less than unity are required.

Now we turn to the photocurrent enhancement observed at high frequencies in several samples. Our results, in particular the polarization dependence for unchanged coefficients $A$ for front and back illumination reveal that this photocurrent is also caused by the linear photogalvanic effect in the surface states. The fact that it is characterized by a nonmonotonic frequency dependence and a magnitude by 10 to 100 times larger as compared to the Drude-like photocurrent clearly indicates that other types of optical transitions must be responsible for the photocurrent formation. This could be either direct optical transitions from the Dirac cone to the bulk states (photoionization) or interband transitions within the Dirac cone.

Direct optical transitions induced by linearly polarized radiation can give rise to a photocurrent in systems of sufficiently low spatial symmetry (piezoelectric class) [69]. An example of such systems is the trigonal surface of topological insulators studied here. Two contributions to the photocurrent, which are of different microscopic origin, are possible. First, the photocurrent can originate from the shift of electron wave packets in the real space at optical transitions (shift contribution) $[69,70]$. Second, the photocurrent can emerge as a result of asymmetric relaxation of the excited electrons/holes. The latter mechanism of the photocurrent formation contains two stages. At the first stage, the optical excitation by linearly polarized radiation leads to an anisotropic distribution of carriers in the momentum space which is described by the stationary correction to the electron distribution function and scales as a square of the $a c$ electric field magnitude. The phenomenon is known in semiconductor physics as the optical alignment of electron momenta $[11,71,72]$. At the second stage, the relaxation of the stationary correction to the distribution function by trigonal scatterers gives rise to a directed flow of carriers, i.e., an electric current. Similar two-step mechanisms of the photocurrent formation have been considered for the surface photocurrents in metals [73] and bulk GaAs [74], as well as for quantum well structures [75].

In the idealized pure linear dispersion model, the photoionization, which results in the depopulation of Dirac states and population of excited bulk states, optical transitions and, consequently, related photocurrents are excited in the range provided by $\hbar \omega>E_{\mathrm{c}}-E_{\mathrm{F}}$. This is because the photoionization picture requires that the initial states of the direct optical transitions are occupied. Furthermore, to excite such transitions the final states must be empty. Therefore these transitions take place only in a certain range of photon energies [23]. In real structures, the dispersion is more complicated and conditions are not as straightforward. That is particularly the case in such samples as our heterostructures combining two different materials. Nevertheless, the photocurrent due to photoionization must show a nonmonotonic resonantlike frequency dependence as observed in experiments, see Figs. 2 and 3(a). According to band structure calculations, the above condition is fulfilled for photon energies corresponding to the enhanced photocurrent in all three $\mathrm{Bi}_{2} \mathrm{Te}_{3} / \mathrm{Sb}_{2} \mathrm{Te}_{3}$ heterostruc- tures and the $\left(\mathrm{Bi}_{0.06} \mathrm{Sb}_{0.94}\right)_{2} \mathrm{Te}_{3}$ sample. Therefore, the model is relevant for the experiments. While this qualitative description of the resonance seems to be appropriated, a theory is not yet developed and is crucially needed. In the $\left(\mathrm{Bi}_{0.57} \mathrm{Sb}_{0.43}\right)_{2} \mathrm{Te}_{3}$ sample the Fermi energy is substantially above the edge of the conduction band, which results in the hybridization of the surface states and excludes photoionization. We attribute the observed resonance in this sample to surface photocurrents served by bulk carriers [74].

Assuming again ideal Dirac fermion bands, interband transitions become possible for photon energies larger than the double Fermi energy. Such systems are characterized by a constant probability as experimentally shown for graphene, where a value of $2.3 \%$ of the optical absorption has been reported [76]. It seems thus unlikely that such transitions are the origin of the observed resonantlike photocurrent.

In experiments in pure $\mathrm{Bi}_{2} \mathrm{Te}_{3}$ no deviation from the Drudelike behavior was found, see Fig. 2(a). Band structure calculations for our samples demonstrate that in $\mathrm{Bi}_{2} \mathrm{Te}_{3}$ samples with $E_{\mathrm{F}}=500 \mathrm{meV}$ direct optical transitions are prohibited in the whole range of used photon energies (up to $250 \mathrm{meV}$ ).

Finally we discuss the circular photogalvanic effect (CPGE) detected at oblique incidence, see Fig. 4. The observed polarization dependence as well as the dependence on the angle of incidence are in full agreement with the phenomenological theory of the CPGE in the surface states, which are characterized by the $\mathrm{C}_{3 \mathrm{v}}$ point group symmetry. The corresponding photocurrent for the $(y z)$ plane of incidence is given by $[52,61,69]$

$$
J_{x}^{\text {circ }}(\varphi)=\gamma t_{\mathrm{p}} t_{\mathrm{s}} E_{0}^{2} P_{\text {circ }} n \sin \theta=C(f, \theta) I \sin 2 \varphi,
$$

in which $\gamma$ is the CPGE constant, $E_{0}$ is the electric field amplitude in vacuum, $t_{\mathrm{p}}$ and $t_{\mathrm{s}}$ are transmission coefficients after Fresnel's formula for linear $p$ and $s$ polarizations, and $n$ is the refraction index. Alike the LPGE resonance addressed above, we attribute the observed CPGE to the photoionization of the surface states [77]. The microscopic mechanism, however, needs to take into account selective excitation of spin branches by circularly polarized radiation which follows from the selection rules. Such processes have been considered previously for 3D TIs excited with near infrared radiation [10] and for 2D TIs excitation of electrons from helical edge states to bulk conduction band states $[20,21,23]$. To conclude on the mechanism responsible for the circular photocurrent observed in our experiments further measurements are required, in particular, a detailed study of the frequency dependencies of the CPGE is needed. This is a subject of future work and is out of scope of the current paper.

\section{CONCLUSION}

To summarize, extensive investigation of the spectra of the photocurrent excited at normal incidence demonstrated that in very different samples and in a wide range of terahertz frequencies it is caused by the linear photogalvanic effect at Drude-like free carrier absorption. These experiments show that spectral studies of the linear photogalvanic effect in the terahertz/microwave range allow one to measure the mobility of the surface states carriers. We emphasize that the photogalvanic effect can only be excited in noncentrosymmetric surface 
states. Thus the frequency behavior of the Drude conductivity can be studied even at room temperature and in materials with substantial conductance in the bulk, where conventional surface electron transport cannot be applied. Besides the LPGE caused by Drude absorption we also observed an enhanced linear photogalvanic effect and the circular photogalvanic effect excited by infrared radiation, which are attributed to the "ionization" of surface states at high frequencies.

\section{ACKNOWLEDGMENTS}

We thank L. E. Golub, M. V. Durnev, and S. A. Tarasenko for fruitful discussions. We are grateful to P. Michel and the ELBEteam for their dedicated support. The support from the DFG priority programmes SFB 1277 (project A04) and SPP1666, the Helmholtz Virtual Institute for Topological Insulators (Jülich-Aachen-Würzburg-Shanghai), and the Elite Network of Bavaria (K-NW-2013-247) is gratefully acknowledged.
[1] M. Z. Hasan and C. L. Kane, Rev. Mod. Phys. 82, 3045 (2010).

[2] X. L. Qi and S. C. Zhang, Rev. Mod. Phys. 83, 1057 (2011).

[3] Y. Ando, J. Phys. Soc. Jpn. 82, 102001 (2013).

[4] A. H. Castro Neto, F. Guinea, N. M. R. Peres, K. S. Novoselov, and A. K. Geim, Rev. Mod. Phys. 81, 109 (2009).

[5] Y. Xia, D. Qian, D. Hsieh, L. Wray, A. Pal, H. Lin, A. Bansil, D. Grauer, Y. S. Hor, R. J. Cava, and M. Z. Hasan, Nat. Phys. 5, 398 (2009).

[6] J. H. Bardarson and J. E. Moore, Rep. Prog. Phys. 76, 056501 (2013).

[7] M. M. Glazov and S. D. Ganichev, Phys. Rep. 535, 101 (2014).

[8] P. Hosur, Phys. Rev. B 83, 035309 (2011).

[9] Q. S. Wu, S. N. Zhang, Z. Fang, and X. Dai, Physica E (Amsterdam) 44, 895 (2012).

[10] J. W. McIver, D. Hsieh, H. Steinberg, P. Jarillo-Herrero, and N. Gedik, Nat. Nanotechnol. 7, 96 (2012).

[11] P. Olbrich, L. E. Golub, T. Herrmann, S. N. Danilov, H. Plank, V. V. Bel'kov, G. Mussler, Ch. Weyrich, C. M. Schneider, J. Kampmeier, D. Grützmacher, L. Plucinski, M. Eschbach, and S. D. Ganichev, Phys. Rev. Lett. 113, 096601 (2014).

[12] J. Duan, N. Tang, X. He, Y. Yan, S. Zhang, X. Qin, X. Wang, X. Yang, F. Xu, Y. Chen, W. Ge, and B. Shen, Sci. Rep. 4, 4889 (2014).

[13] A. Junck, G. Refael, and F. von Oppen, Phys. Rev. B 90, 245110 (2014).

[14] K.-M. Dantscher, D. A. Kozlov, P. Olbrich, C. Zoth, P. Faltermeier, M. Lindner, G. V. Budkin, S. A. Tarasenko, V. V. Bel'kov, Z. D. Kvon, N. N. Mikhailov, S. A. Dvoretsky, D. Weiss, B. Jenichen, and S. D. Ganichev, Phys. Rev. B 92, 165314 (2015).

[15] S. Y. Hamh, S.-H. Park, S.-K. Jerng, J. H. Jeon, S.-H. Chun, and J. S. Lee, Phys. Rev. B 94, 161405(R) (2016).

[16] K. N. Okada, N. Ogawa, R. Yoshimi, A. Tsukazaki, K. S. Takahashi, M. Kawasaki, and Y. Tokura, Phys. Rev. B 93, 081403(R) (2016).

[17] H. Plank, L. E. Golub, S. Bauer, V. V. Bel'kov, T. Herrmann, P. Olbrich, M. Eschbach, L. Plucinski, C. M. Schneider, J. Kampmeier, M. Lanius, G. Mussler, D. Grützmacher, and S. D. Ganichev, Phys. Rev. B 93, 125434 (2016).

[18] H. Plank, S. N. Danilov, V. V. Bel'kov, V. A. Shalygin, J. Kampmeier, M. Lanius, G. Mussler, D. Grützmacher, and S. D. Ganichev, J. Appl. Phys. 120, 165301 (2016).

[19] Y. Pan, Q.-Z. Wang, A. L. Yeats, T. Pillsbury, T. C. Flanagan, A. Richardella, H. Zhang, D. D. Awschalom, C.-X. Liu, and N. Samarth, Nat. Commun. 8, 1037 (2017).
[20] V. Kaladzhyan, P. P. Aseev, and S. N. Artemenko, Phys. Rev. B 92, 155424 (2015).

[21] L. I. Magarill and M. V. Entin, JETP Lett. 104, 771 (2016).

[22] M. V. Entin and L. I. Magarill, JETP Lett. 103, 711 (2016).

[23] K.-M. Dantscher, D. A. Kozlov, M.-T. Scherr, S. Gebert, J. Bärenfänger, M. V. Durnev, S. A. Tarasenko, V. V. Bel'kov, N. N. Mikhailov, S. A. Dvoretsky, Z. D. Kvon, J. Ziegler, D. Weiss, and S. D. Ganichev, Phys. Rev. B 95, 201103(R) (2017).

[24] D. A. Bas, K. Vargas-Velez, S. Babakiray, T. A. Johnson, P. Borisov, T. D. Stanescu, D. Lederman, and A. D. Bristow, Appl. Phys. Lett. 106, 041109 (2015).

[25] D. A. Bas, R. A. Muniz, S. Babakiray, D. Lederman, J. E. Sipe, and A. D. Bristow, Opt. Express 24, 23583 (2016).

[26] C. Kastl, T. Guan, X. Y. He, K. H. Wu, Y. Q. Li, and A. W. Holleitner, Appl. Phys. Lett. 101, 251110 (2012).

[27] Ch. Kastl, Ch. Karnetzky, H. Karl, and A. W. Holleitner, Nat. Commun. 6, 6617 (2015).

[28] L. Braun, G. Mussler, A. Hruban, M. Konczykowski, M. Wolf, T. Schumann, M. Münzenberg, L. Perfetti, and T. Kampfrath, Nat. Commun. 7, 13259 (2016).

[29] C. Kastl, C. Karnetzky, A. Brenneis, F. Langrieger, and A. Holleitner, IEEE J. Sel. Top. Quantum Electron. 23, 8700305 (2017).

[30] P. Seifert, K. Vaklinova, S. Ganichev, K. Kern, M. Burghard, and A. W. Holleitner, Nat. Commun. 9, 331 (2018).

[31] K. Kuroda, J. Reimann, J. Güdde, and U. Höfer, Phys. Rev. Lett. 116, 076801 (2016).

[32] A. M. Shikin, I. I. Klimovskikh, M. V. Filyanina, A. A. Rybkina, D. A. Pudikov, K. A. Kokh, and O. E. Tereshchenko, Phys. Solid State 58, 1675 (2016).

[33] K. Kuroda, J. Reimann, K. A. Kokh, O. E. Tereshchenko, A. Kimura, J. Güdde, and U. Höfer, Phys. Rev. B 95, 081103(R) (2017).

[34] A. M. Shikin, V. Yu. Voroshin, A. G. Rybkin, K. A. Kokh, O. E. Tereshchenko, Y. Ishida, and A. Kimura, 2D Materials 5, 015015 (2018).

[35] I. Garate and M. Franz, Phys. Lett. 104, 146802 (2010).

[36] D. Hsieh, J. W. McIver, D. H. Torchinsky, D. R. Gardner, Y. S. Lee, and N. Gedik, Phys. Rev. Lett. 106, 057401 (2011).

[37] D. Hsieh, F. Mahmood, J. W. McIver, D. R. Gardner, Y. S. Lee, and N. Gedik, Phys. Rev. Lett. 107, 077401 (2011).

[38] J. W. McIver, D. Hsieh, S. G. Drapcho, D. H. Torchinsky, D. R. Gardner, Y. S. Lee, and N. Gedik, Phys. Rev. B 86, 035327 (2012).

[39] E. L. Ivchenko and S. D. Ganichev, arXiv:1710.09223. 
[40] L. Plucinski, G. Mussler, J. Krumrain, A. Herdt, S. Suga, D. Grützmacher, and C. M. Schneider, Appl. Phys. Lett. 98, 222503 (2011).

[41] J. Kampmeier, S. Borisova, L. Plucinski, M. Luysberg, G. Mussler, and D. Grützmacher, Cryst. Growth Des. 15, 390 (2015).

[42] M. Eschbach, E. Mlynczak, J. Kellner, J. Kampmeier, M. Lanius, E. Neumann, C. Weyrich, M. Gehlmann, P. G. S. Döring, G. Mussler, N. Demarina, M. Luysberg, G. Bihlmayer, Th. Schäpers, L. Plucinski, S. Blügel, M. Morgenstern, C. M. Schneider, and D. Grützmacher, Nat. Commun. 6, 8816 (2015).

[43] M. Lanius, J. Kampmeier, C. Weyrich, S. Kölling, M. Schall, P. Schüffelgen, E. Neumann, M. Luysberg, G. Mussler, P. M. Koenraad, T. Schäpers, and D. Grützmacher, Cryst. Growth Des. 16, 2057 (2016).

[44] J. Kellner, M. Eschbach, J. Kampmeier, M. Lanius, E. Mlynczak, G. Mussler, B. Holländer, L. Plucinski, M. Liebmann, D. Grützmacher, C. M. Schneider, and M. Morgenstern, Appl. Phys. Lett. 107, 251603 (2015).

[45] C. Weyrich, M. Drögeler, J. Kampmeier, M. Eschbach, G. Mussler, T. Merzenich, T. Stoica, I. E. Batov, J. Schubert, L. Plucinski, B. Beschoten, C. M. Schneider, C. Stampfer, D. Grützmacher, and Th. Schäpers, J. Phys.: Condens. Matter 28, 495501 (2016).

[46] S. D. Ganichev, W. Prettl, and P. G. Huggard, Phys. Rev. Lett. 71, 3882 (1993).

[47] P. Schneider, J. Kainz, S. D. Ganichev, V. V. Bel'kov, S. N. Danilov, M. M. Glazov, L. E. Golub, U. Rössler, W. Wegscheider, D. Weiss, D. Schuh, and W. Prettl, J. Appl. Phys. 96, 420 (2004).

[48] P. Michel, R. W. Dekorsy, P. Evtushenko, F. G. Gabriel, E. G. Grosse, M. H. Helm, M. K. Krenz, U. Lehnert, W. Seidel, D. W. Wohlfarth, A. W. Wolf, and R. W. Wünsch, in Proceeding of the International Free Electron Laser Conference FEL 2004, ELETTRA, Trieste, Italy, online at http://accelconf.web.cern.ch/ AccelConf/f04/papers/MOAIS04/MOAIS04.PDF.

[49] P. Michel, H. Büttig, F. Gabriel, M. Helm, U. Lehnert, Ch. Schneider, R. Schurig, W. Seidel, D. Stehr, J. Teichert, S. Winnerl, and R. Wünsch, in Proceeding of the International Free Electron Laser Conference FEL 2006, BESSY, Berlin, Germany, online at http://accelconf.web.cern.ch/AccelConf/ f06/PAPERS/TUCAU02.PDF.

[50] J. Faist, F. Capasso, D. L. Sivco, C. Sirtori, A. L. Hutchinson, and A. Y. Cho, Science 264, 553 (1994).

[51] J. Faist, F. Capasso, C. Sirtory, D. L. Sivko, and A. Y. Cho, in Quantum Cascade Lasers, Series Semiconductors and Semimetals, edited by R. K. Willardson and E. R. Weber, Vol. 66, Intersubband Transitions in Quantum Wells, Volume edited by H. C. Liu and F. Capasso (Academic Press, San Diego, 2000).

[52] S. D. Ganichev and W. Prettl, Intense Terahertz Excitation of Semiconductors (Oxford University Press, Oxford, 2006).

[53] S. D. Ganichev, S. A. Emel'yanov, and I. D. Yaroshetskii, Pisma Zh. Eksp. Teor. Fiz. 35, 297 (1982) [JETP Lett. 35, 368 (1982)].

[54] C. Jiang, V. A. Shalygin, V. Y. Panevin, S. N. Danilov, M. M. Glazov, R. Yakimova, S. Lara-Avila, S. Kubatkin, and S. D. Ganichev, Phys. Rev. B 84, 125429 (2011).

[55] S. D. Ganichev, E. Ziemann, Th. Gleim, W. Prettl, I. N. Yassievich, V. I. Perel, I. Wilke, and E. E. Haller, Phys. Rev. Lett. 80, 2409 (1998).
[56] V. Lechner, L. E. Golub, P. Olbrich, S. Stachel, D. Schuh, W. Wegscheider, V. V. Bel'kov, and S. D. Ganichev, Appl. Phys. Lett. 94, 242109 (2009) [47]; S. D. Ganichev, Petra Schneider, V. V. Bel'kov, E. L. Ivchenko, S. A. Tarasenko, W. Wegscheider, D. Weiss, D. Schuh, B. N. Murdin, P. J. Phillips, C. R. Pidgeon, D. G. Clarke, M. Merrick, P. Murzyn, E. V. Beregulin, and W. Prettl, Phys. Rev. B 68, 081302(R) (2003).

[57] S. Dvoretsky, N. Mikhailov, Y. Sidorov, V. Shvets, S. Danilov, B. Wittman, and S. D. Ganichev, J. Electron. Mater. 39, 918 (2010).

[58] S. D. Ganichev, Y. V. Terent'ev, and I. D. Yaroshetskii, Pisma Zh. Tekh. Fiz. 11, 46 (1985) [Sov. Tech. Phys. Lett. 11, 20 (1989)].

[59] E. Ziemann, S. D. Ganichev, I. N. Yassievich, V. I. Perel, and W. Prettl, J. Appl. Phys. 87, 3843 (2000).

[60] C. Drexler, N. Dyakonova, P. Olbrich, J. Karch, M. Schafberger, K. Karpierz, Yu. Mityagin, M. B. Lifshits, F. Teppe, O. Klimenko, Y. M. Meziani, W. Knap, and S. D. Ganichev, J. Appl. Phys. 111, 124504 (2012).

[61] S. D. Ganichev and W. Prettl, topical review, J. Phys.: Condens. Matter 15, R935 (2003).

[62] M. P. Walser, U. Siegenthaler, V. Lechner, D. Schuh, S. D. Ganichev, W. Wegscheider, and G. Salis, Phys. Rev. B 86, 195309 (2012).

[63] M. Kohda, V. Lechner, Y. Kunihashi, T. Dollinger, P. Olbrich, C. Schönhuber, I. Caspers, V. V. Bel'kov, L. E. Golub, D. Weiss, K. Richter, J. Nitta, and S. D. Ganichev, Phys. Rev. B 86, 081306(R) (2012).

[64] B. E. A. Saleh and M. C. Teich, Fundamentals of Photonics (John Wiley \& Sons, Inc., Hoboken, New Jersey, 2007).

[65] V. V. Bel'kov, S. D. Ganichev, E. L. Ivchenko, S. A. Tarasenko, W. Weber, S. Giglberger, M. Olteanu, H.-P. Tranitz, S. N. Danilov, P. Schneider, W. Wegscheider, D. Weiss, and W. Prettl, J. Phys.: Condens. Matter 17, 3405 (2005).

[66] This is also true if the Fermi energy is larger than the band gap. ARPES measurements presented in Ref. [17] show that the surface states are well described by the linear dispersion even at such rather high energies, see Figs. 1(a) and 1(c) obtained, respectively, for samples $\mathrm{Bi}_{2} \mathrm{Te}_{3}$ and $\left(\mathrm{Bi}_{0.57} \mathrm{Sb}_{0.43}\right)_{2} \mathrm{Te}_{3}$ used in the present study. At a Fermi level greater than the band gap electrons in the bulk as well as electrons in the surface states contribute to linear electron transport and the sample conductivity is dominated by the bulk electrons. Free carrier absorption, or high frequency Drude conductivity, is caused by intraband indirect optical transitions. For small photon energies initial states of these transitions lie slightly below the Fermi level. The bulk states, however, do not contribute to the studied photogalvanic effects because it is forbidden in the bulk due to symmetry reasons.

[67] Note that the highest mobility value, extracted for $\left(\mathrm{Bi}_{0.06} \mathrm{Sb}_{0.94}\right)_{2} \mathrm{Te}_{3}$, corresponds, at first glance surprisingly, to the shortest scattering time. This is due to the very small value of the Fermi energy obtained from ARPES and in fact can be overestimated.

[68] T. P. Ginley, Y. Wang, and S. Law, Crystals 6, 154 (2016).

[69] E. L. Ivchenko, Optical Spectroscopy of Semiconductor Nanostructures (Alpha Science, Harrow, 2005).

[70] B. I. Sturman and V. M. Fridkin, The Photovoltaic and Photorefractive Effects in Noncentrosymmetric Materials (Gordon and Breach, Philadelphia, 1992). 
[71] D. N. Mirlin, in Optical Orientation, edited by F. Meier and B. P. Zakharchenya (Elsevier Science, Amsterdam, 1984).

[72] L. E. Golub, S. A. Tarasenko, M. V. Entin, and L. I. Magarill, Phys. Rev. B 84, 195408 (2011).

[73] L. I. Magarill and M. V. Entin, Zh. Eksp. Teor. Fiz. 81, 1001 (1981) [Sov. Phys. JETP 54, 531 (1981)]

[74] V. L. Alperovich, V. I. Belinicher, V. N. Novikov, and A. S. Terekhov, Ferroelectrics 45, 1 (1982).
[75] S. A. Tarasenko, Phys. Rev. B 83, 035313 (2011).

[76] R. R. Nair, P. Blake, A. N. Grigorenko, K. S. Novoselov, T. J. Booth, T. Stauber, N. M. R. Peres, and A. K. Geim, Science 320, 1308 (2008).

[77] Note that in some materials, apart from topological surface states, Rashba-Dresselhaus spin-split states may also contribute to the photogalvanic effects. 